\title{
A Model Calculation on Optical Gain and Co-Stimulated Emissions of Photons and Phonons in Silicon
}

\author{
M.J. Chen ${ }^{1}$ and C.S. Tsai ${ }^{2,3}$ \\ ${ }^{1}$ Dept. of Materials Science and Eng., National Taiwan University, Taipei, Taiwan 106 \\ ${ }^{2}$ Dep.t of Electrical Eng. and Computer Science, University of California, Irvine, CA 92697, USA \\ ${ }^{3}$ Institute of Electro-Optical Eng., National Taiwan University, Taipei, Taiwan 106
}

Group IV materials-based micro- and nano-photonics has gradually become a very active area for research worldwide. ${ }^{1}$ A variety of passive and active components and devices have been explored and/or realized. ${ }^{1}$ For the key component of silicon-based light sources, numerous approaches, mostly involving the experimental studies, have been explored with significant progresses. $^{2-23}$ On the theoretical study of the subject, optical gain in materials with indirect transitions such as bulk crystalline silicon was reassessed recently based on a simple two-level model. ${ }^{24}$ In this paper, we present a model calculation on the optical gain at bandgap energy and the rate equations for electron, photon, and phonon in silicon which takes into account the detailed band-edge structures of the conduction and valence bands.

The theoretical analysis has arrived at an expression for optical gain $g(\hbar \omega)$ via phonon-assisted optical transitions in indirect bandgap semiconductors such as silicon as follows

$g(\hbar \omega)=\frac{h^{3} c^{2}}{8 \pi n^{2}(\hbar \omega)^{2}} R_{s p}(\hbar \omega) \cdot\left\{1-n_{q} /\left(n_{q}+1\right) \cdot \exp \left[(\hbar \omega+\hbar \Omega-\Delta F) / k_{B} T\right]\right\}$

where $R_{s p}(\hbar \omega)$ is the spontaneous emission rate, $n_{q}$ is the phonon occupation number, $\hbar \omega$ and $\hbar \Omega$ are the photon and phonon energies, $\hbar$ is the reduced Planck constant, $c$ is the velocity of light in free space, $n$ is the refractive index, $\Delta F$ is the difference of the quasi-Fermi levels for electrons and holes, $k_{B}$ is the Boltzmann constant, and $T$ is the temperature. It is seen that the sign of $g(\hbar \omega)$ is determined by $\Delta F$ and its magnitude is also proportional to $R_{s p}(\hbar \omega)$. Various carrier localization structures such as nanostructured $\mathrm{PN}$ junctions ${ }^{12}$ may be utilized to enhance $R_{s p}(\hbar \omega)$ and thus $g(\hbar \omega)$. Clearly, an optical gain can take place if the quantity in braces is positive, i.e.

$\left(n_{q}+1\right) / n_{q}>\exp \left[(\hbar \omega+\hbar \Omega-\Delta F) / k_{B} T\right]$

Figure 1 shows the relationship between $\Delta F$ and $n_{q}$ required for population inversion involving transverse optical (TO) phonon ( $\hbar \Omega=57.8 \mathrm{meV}$ )-assisted optical transition at photon energy $\hbar \omega=1.07 \mathrm{eV}$ in crystalline silicon at $300 \mathrm{~K}$. The solid curve in Fig. 1 depicts the condition and the region for population inversion. The phonon occupation number at thermodynamic equilibrium $\mathrm{n}_{\mathrm{q} 0}$ and the condition $\Delta F=\hbar \omega+\hbar \Omega$ are indicated in dotted lines, respectively. The region above the solid curve is the positive optical gain region $(g(\hbar \omega)>0)$, while the region below the solid curve is the absorption region $(g(\hbar \omega)<0)$.

Since a phonon is emitted during the stimulated emission of a photon and since a phonon is absorbed during the absorption of a photon, a net stimulated emission of phonons will occur when the population inversion is reached. Therefore, the condition for the net stimulated phonon emission resulting from the net stimulated emission of photons with energy $\hbar \omega$ is the same as Eq.(2). The increase in the phonon population due to the net stimulated phonon emission results in a deviation of phonon occupation number $n_{q}$ from its value at thermal equilibrium. Figure 1 shows that as the phonon occupation number $n_{q}$ increases from the value at thermodyamic equilibrium $n_{q 0}$, a larger value of $\Delta F$ is required to facilitate population inversion. 


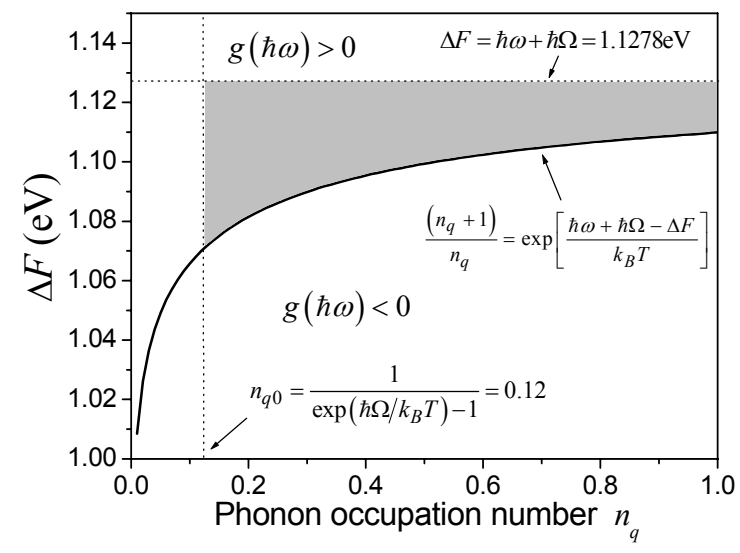

Figure 1: Relationship between $\Delta F$ and $n_{q}$ required for population inversion in bulk crystalline silicon at $300 \mathrm{~K}$.

The net generation rates for photon and phonon are derived as follows $R_{s t}(\hbar \omega)-R_{a b}(\hbar \omega)+R_{s p}(\hbar \omega)$

$$
=M \cdot\left[\left(n_{p}+1\right)\left(n_{q}+1\right)-n_{p} n_{q} \exp \left(\frac{\hbar \omega+\hbar \Omega-\Delta F}{k_{B} T}\right)\right] \cdot N P
$$

where $R_{s t}(\hbar \omega), R_{s p}(\hbar \omega)$ and $R_{a b}(\hbar \omega)$ are, respectively, the rates of stimulated emission, spontaneous emission and absorption; $n_{p}$ is the photon occupation number; $M$ is a constant related to radiative recombination; and $N$ and $P$ are the electron and hole concentrations. Note that in Eq.(3), the term $\left(n_{p}+1\right)\left(n_{q}+1\right)$ corresponds to the stimulated and spontaneous emission rates of photon and phonon, while the term $n_{p} n_{q} \exp \left[(\hbar \omega+\hbar \Omega-\Delta F) / k_{B} T\right]$ represents the absorption rate of photon and phonon.

The rate equations for electron, photon and phonon involved are given by

$\frac{d N}{d t}=R_{p}-M n_{p}\left[\left(n_{q}+1\right)-n_{q} \exp \left(\frac{\hbar \omega+\hbar \Omega-\Delta F}{k_{B} T}\right)\right] N^{2}-M\left(n_{q}+1\right) N^{2}-\frac{N}{\tau_{c}}$

$\frac{d n_{p}}{d t}=\frac{M}{K_{p}} n_{p}\left[\left(n_{q}+1\right)-n_{q} \exp \left(\frac{\hbar \omega+\hbar \Omega-\Delta F}{k_{B} T}\right)\right] N^{2}+\beta \frac{M}{K_{p}}\left(n_{q}+1\right) N^{2}-\frac{n_{p}}{\tau_{p}}$

$\frac{d n_{q}}{d t}=\frac{M}{K_{q}} n_{p}\left[\left(n_{q}+1\right)-n_{q} \exp \left(\frac{\hbar \omega+\hbar \Omega-\Delta F}{k_{B} T}\right)\right] N^{2}+\frac{M}{K_{q}}\left(n_{q}+1\right) N^{2}-\frac{n_{q}-n_{q 0}}{\tau_{q}}$

where $R_{p}$ is the pumping rate either by current injection or optical excitation; $\beta$ is a factor which represents the fraction of spontaneous emission entering the optical mode; $\tau_{c}, \tau_{p}$ and $\tau_{q}$ are the lifetimes of carrier, photon and phonon, respectively; and $K_{p}$ and $K_{q}$ are the density of states for photon and phonon, respectively.

We set $d / d t=0$ in Eqs.(4a) (4c) and solve for the steady state solution of the rate equations. The threshold condition for laser oscillation is then obtained as follows

$M\left[\left(n_{q}+1\right)-n_{q} \exp \left(\frac{\hbar \omega+\hbar \Omega-\Delta F}{k_{B} T}\right)\right] N^{2}=\frac{K_{p}}{\tau_{p}}$

Eq.(5) shows that the photon loss of the resonant cavity must be compensated by the optical gain for the on-set of laser oscillation. The steady state solutions for electrons, photons, and phonons above the threshold are 


$$
\begin{aligned}
& N \approx \sqrt{\frac{K_{p} / \tau_{p}}{M\left\{n_{q 0}\left[1-\exp \left(\frac{\hbar \omega+\hbar \Omega-\Delta F}{k_{B} T}\right)\right]+1\right\}}} \equiv N_{t h} \\
& N_{q} \approx N_{q 0}+\tau_{q} R_{p} \\
& N_{p} \approx \tau_{p}\left(R_{p}-R_{p t h}\right)
\end{aligned}
$$

where $N_{p}=K_{p} n_{q}$ and $N_{q}=K_{q} n_{q}$ are, respectively, the photon density and the phonon density, $N_{q 0}=K_{q} n_{q 0}$ is the phonon density at thermodynamic equilibrium, and $R_{p t h}$ is the pumping rate at threshold. Below the threshold, the photon density $N_{p}$ increases rather slowly. However, once the threshold is reached, the photon density $N_{p}$ increases rapidly and linearly with the pumping rate $R_{p}$. As to the phonon, its density $N_{q}$ remains practically constant before threshold, but increases linearly with the pumping rate $R_{p}$ once the threshold is reached. Both the photon and the phonon densities grow rapidly after the threshold is reached, which indicates the co-stimulated emission of photons and phonons. On the other hand, the carrier concentration $N$ increases linearly with the pumping rate $R_{p}$ before the threshold is reached, but clamps at its threshold value $N_{t h}$ after the threshold is reached.

In summary, the approach and findings of a model calculation on the optical gain at bandgap energy and the solution of the rate equations for electron, photon and phonon in indirect bandgap semiconductors such as silicon are presented.

\section{REFERENCE}

${ }^{1}$ See, for example, R.A Soref, Proc. IEEE 81, 1687 (1993); W.X. Ni, editor, Silicon-Based Optoelectronics: Advances and Future Perspectives, (Elsevier, 2003); L. Pavesi and D. J. Lockwood (eds), Silicon Photonics, Topics in Applied Physics, Vol.94 (Springer-Verlag, New York, 2004); the plenary papers by S.A. Lynch and D.J. Paul; M. Paniccia and G. Pomrenke; and the invited papers by P. Boucaud et al; N. Usami et al; K. Kawaguchi and Y. Shiraki; J. Yu; S. Matthias et al, and J. Lee and J.H. Shin at $1^{\text {st }}$ Int. Conf. on Group IV Photonics, Sept. 29-Oct. 1, 2004, Hong Kong.

${ }^{2}$ L. Pavesi et al, Nature 408, 440 (2000), and K. Luterova et al, Optical Materials, 27, 750 (2005).

${ }^{3}$ L. Tsybeskov et al., Phys. Rev. B 54, R8361 (1996), and P. M. Fauchet et al, Opt. Materials, 27, 745 (2005).

${ }^{4}$ Z.H. Lu, D.J. Lockwood and J.M. Baribeau, Nature 378, 258 (1995).

${ }^{5}$ L.T.Canham, Appl. Phys. Lett. 57, 1046 (1990).

${ }^{6}$ P.M. Fauchet, IEEE J. Select. Topics Quantum Electron. 4, 1020 (1998).

${ }^{7}$ J. Palm, F. Gan, B. Zheng, J. Michel, and L. C. Kimerling, Phys. Rev. B 54, 17603 (1996); J. Michel, et al., Mater. Res. Soc. Symp. Proc. 442, 317 (1996).

${ }^{8}$ S. Coffa and G. Franzo, Appl. Phys. Lett. 69, 2077 (1996); A. Polman, J. Appl. Phys. 82, 1, (1997).

${ }^{9}$ L. Tsybeskov, et al., Appl. Phys. Lett. 69, 3411 (1996).

${ }^{10}$ W. L. Ng, M. A. Louren, R. M. Gwilliam, S. Ledain, G. Shao, K. P. Homewood, Nature 410, 192 (2001).

${ }^{11}$ M. A. Green, J. Zhao, A. Wang, P. J. Reece, M. Gal, Nature 412, 805 (2001).

${ }^{12}$ M. J. Chen, J. L. Yen, J. Y. Li, J. F. Chang, S. C. Tsai and C. S. Tsai, Appl. Phys. Lett. 84, 2163 (2004).

${ }^{13}$ H.A. Atwater, G. I. Bourianoff, and R.J. Walters, Nature Materials 4, 143 (2005)

${ }^{14}$ M. Zacharias, et al., Appl. Phys. Lett. 80, 661 (2002).

${ }^{15}$ A. Polman, et al., Appl. Phys. Lett. 84, 1037 (2004).

${ }^{16}$ L. Tsybeskov, et al., Phys. Stat. Sol. (a) 165, 69 (1998).

${ }^{17}$ J. Faist, et al., Science, 264, 5530556 (1994); G. Dehlinger, et al., Science, 290, 2277 (2000).

${ }^{18}$ M.H. Nayfeh et al, Appl. Phys. Lett., 80, 121 (2002).

${ }^{19}$ L. Dal Negro et al., J. Appl. Phys., 96, 5747 (2004).

${ }^{20}$ L. Khriachtchev et al., Appl. Phys. Lett., 85, 1511 (2004).

${ }^{21}$ V.R. Almeida, C.A. Barrios, R.R. Panepucci, and M. Lipson, Nature, 1081 (Oct, 2004).

${ }^{22}$ O. Boyraz and B. Jalali, Optics Express, 12, 5269 (2004).

${ }^{23}$ H. Rong, R. Jones, A. Liu, O. Cohen, D. Hak, A. Fang and M. Paniccia, Nature 433, 725 (2005).

${ }^{24}$ T. Trupke, M. A. Green and P. Würfel, J. Appl. Phys. 93, 9058 (2003). 